\title{
Probability and Tempered Modal Eliminativism
}

\author{
Michael J. Shaffer \\ Department of Philosophy, Southern Connecticut, State University, 501 Crescent Street, New Haven, \\ CT 06515, USA
}

Received 16 October 2003 Accepted 22 February 2004 Revised 4 March 2004

In this paper the strategy for the eliminative reduction of the alethic modalities suggested by John Venn is outlined and it is shown to anticipate certain related contemporary empiricistic and nominalistic projects. Venn attempted to reduce the alethic modalities to probabilities, and thus suggested a promising solution to the nagging issue of the inclusion of modal statements in empiricistic philosophical systems. However, despite the promise that this suggestion held for laying the 'ghost of modality' to rest, this general approach, tempered modal eliminativism, is shown to be inadequate for that task.

\section{Tempered modal eliminativism}

Many philosophers and logicians have suggested that there is a close tie between the concepts of possibility and of probability. It is also true that many philosophers have regarded the modalities with deep suspicion, especially in the context of the sciences. ${ }^{1}$ Van Fraassen comments that,

in the Aristotelian tradition, natural philosophy was deeply involved with modality: necessity, possibility, contingency, potentiality. Nominalism and modern empiricism rejected this role of modality, arguing that necessity, for example, attaches only to the relations among ideas, or among words, and not to physical occurrences. But, empiricism has not been lucky with modality; as Herman Weyl said, the ghost of modality is not easily laid. In this century the problem has become much more acute, for a new modality, a possibility-with-degrees, has taken center stage in physical science: probability. (1980, p. 158.)

However, the 'ghost of modality' that nominalists and empiricists find to be so offensive has long been supported by philosophers with rationalist proclivities. In particular, Leibniz was a staunch defender of the legitimacy both of the concept of possibility and of the concept of probability. More interestingly, Leibniz argues that probability is just degree of possibility (i.e. probability can be reduced to degrees of possibility), ${ }^{2}$ and this Leibnizian view will be referred to here as probabilistic eliminativism. ${ }^{3}$ Following Leibniz, one might want to claim that probability is just graded modality, and I presume that the empiricists to

1 Quine's well-known views are a good example of this kind of skepticism with respect to the modalities. See Quine (1960, ch. 3, 1947, 1953) for details.

2 See Beirmann and Faak 1957, Hacking 1971 and Hacking (1975, chs. 13-14).

3 This view constitutes a reductive elimination of probability, and depends on there being an isomorphism between the structure of probabilities (the algebra of probabilities) and the structure of possibilities (the algebra of possibilities), but the mapping from the elements of the algebra of probabilities to the elements of the algebra of possibilities is not a one-to-one mapping. As a result, the algebras are not isomorphic, and, hence, probabilities cannot be reduced to possibilities without loss of meaning. 
which van Fraassen refers would just as well get rid of both concepts if this turns out to be the case as they would then, presumably, both be equally offensive.

However, this radical eliminativist view, extreme modal eliminativism, seems as if it may be rather too extreme even for nominalistic empiricists, especially when one notes the importance of the concept of probability in the contexts of the physical sciences, confirmation theory, decision theory, game theory, as well as a whole host of other important related fields. ${ }^{4}$ Certainly one need not treat probabilities and the traditional modalities in the same manner, and, as Niiniluoto notes in his discussing traditional views of modality and probability,

... the classical theory also suggests that probability theory is in fact reducible to the theory of modality: if the concepts of necessity, possibility, and equipossibility are given, then a mathematical notion of probability is understood as well. (1988a, p. 279.)

A modern, more sophisticated, version of this modality friendly project can be found in Bigelow 1976. In the Leibnizian tradition, Bigelow attempts to provide what, in modern parlance, we might refer to as a possible worlds account of probability, and this is most certainly a modality based account of probability. Bigelow claims that,

Given a numerical measure of the similarities between pairs of worlds, there will be various derivative numerical measures which can be assigned to sets of possible worlds, or propositions. It is possible that some of these numerical measures on propositions might be related to their probabilities. (1976, p. 305.)

He also adds that,

... a measure of the diameter of a proposition gives some indication of the range of possibilities which it encompasses, or the variety of ways in which it can come to be true. We might naturally conjecture that a proposition which is true in widely dissimilar worlds will, in general, be more probable than one in which is true only in more a restricted range of very similar worlds. Consequently, the notion of the diameters of propositions seems to be related to the notion of probability. (1976, p. 305.)

Similarly, in discussing the merit of regarding modalities as dyadic operators, von Wright has suggested that,

Of old, probability and possibility had been regarded as related ideas. What is the probability of a proposition, on given evidence, but the degree to which this proposition, on the evidence is possible? This being so it was tempting to look for a formal similarity of structure also between modal logic and probability theory.

4 The sense in which this program is an eliminative program, is in the sense of elimination via reduction. Probability claims are, in the Leibnizian view, completely eliminable slava veritate and without loss of meaning by systematically replacing them with the more fundamental modal claims in terms of which they are defined. 
Whatever the correct solution in this matter may be, it is possible to construct a formal dyadic modal logic which has the following interesting properties:

1. Monadic modal logic of the traditional type can be viewed as a limiting (or 'degenerate') case of dyadic logic, viz., as a logic of possibility relative to tautologous evidence.

2. Dyadic modal logic can be given an axiomatic presentation which is strikingly similar to an axiomatic presentation of the probability calculus.

The logic of probability can thus be viewed as a numerical modal logic. (1989, pp. 31-2.)

In this passage not only does von Wright indicate both the striking structural similarities between the alethic modalities and the probability calculus and the prima facie plausibility of regarding the probability calculus as a more precise modal logic, but also, he raises the controversial issue whether one might regard the alethic modalities as conditional concepts where the modalities in question are conditional on tautologous evidence. Ignoring the specific details of Bigelow's and von Wright's accounts, we can simply note that, in accord with the Leibnizian doctrine mentioned above, it is not completely implausible that probabilities might be related to the modalities, or to some properties associated with modal concepts (e.g. Bigelow's diameters). ${ }^{5}$

However, neither this kind of project nor extreme modal eliminativism would be acceptable to those nominalistic empiricists who harbor a general skepticism with respect to the modalities, but have no such prejudice with respect to probabilities for the reasons mentioned above. As a result, one might wonder about the acceptability of less extreme forms of modal eliminativism. In accord with this sort of general intuition, Niiniluoto also notes that,

Another, more promising alternative would be to reduce modality to probability. If we could analyse the truth conditions for probability statements without employing modal concepts, then necessity could be defined by probability one, impossibility by probability zero, and possibility by non-zero probability. (1988a, p. 279.)

The empiricist inspired elimination of the traditional modalities mentioned by van Fraassen, and championed by Quine, might then be achieved by reducing the modalities to probabilities via definition. This eliminativist project, tempered modal eliminativism, might then initially appear to be a satisfactory tack for the modal eliminativist to take, and it would both maintain the status of the probability calculus as well as explain the prevalence of modal discourse as parasitic on the prevalence of probabilistic discourse. ${ }^{6}$

Historically, the project of reducing the traditional modalities to probabilities was vigorously pursued by the logician John Venn in The Logic of Chance, (1866, ch. XII),

5 Now, Bigelow is clear that we cannot tell what the probability of a proposition is from the diameter of a proposition alone. Rather, he identifies them with a different technical concept associated with possible worlds; viz. volumes. See Bigelow (1976, pp. 305-8) for more details. See Nilsson 1986 for a variant of this strategy.

6 This might straightforwardly seem to be the case as standard alethic modal operators are non-epistemic unary operators whereas probabilities are epistemic operators that are often conditional and dyadic, and this might indicate that probability is really an epistemic concept that depends on one's evidence and so is not a modality in the normal sense associated with the alethic modalities. 
and as we have seen this view of the modalities has some intuitive appeal, particularly for empiricists. ${ }^{7}$ With this idea in mind, Venn argued that none of the traditional divisions of the modalities in terms of their strength is as precise as the probability calculus, which admits of continuous numerical degrees, ${ }^{8}$ and that,

... the logicians, after having had a long and fair trial, have totally failed to make anything satisfactory out of this subject of the modals by their methods of enquiry and treatment; and that it ought, therefore, to be banished entirely from that science, and relegated to probability. (1866, p. 299.)

So, it seems that the modalities might just be coarse-grained probabilities, which can be more precisely expressed without any loss of meaning in terms of numerical probabilities. With respect to possibility, for example, this intuition seems prima facie plausible as the possible truth of $\mathrm{p}$ seems to be something rather like the probable truth of $\mathrm{p}$, and the probable truth of $\mathrm{p}$ can be given a precise numerical value in the continuous interval $[0,1]$. Specifically, possibility would be identified with the half-open, half-closed, interval $[0,1]$. Similarly, impossibility would be identified with 0 probability, necessity with probability 1, etc. In honor of Venn's seminal contribution on the issue, I will refer to this particular project as the Venn project, and it requires that all modal concepts can be eliminated and replaced by probability statements. As such, the Venn project is a form of tempered modal eliminativism. ${ }^{9}$ The sections that follow will specifically concern the concept of

7 For historical details see Niiniluoto 1988a, Niiniluoto $1988 b$ and Venn 1866.

8 See, especially, Venn (1866, ch. XII, § 20). Venn discusses several early attempts to rank modal concepts from the weakest to strongest, and he argues that such attempts were primitive or coarse modal attempts to do the work of the concept of probability in discrete terms. But, Venn superficially refers to the modalities in terms of degrees of certainty, and, as a result, one might be tempted to regard his project as only aimed at eliminating the epistemic modalities. However, it is clear that elimination of the modalities is intended to extend to the logical modalities and not just to epistemic modalities. Venn is clearly ambiguous about the nature of the modalities themselves as well as about the nature of logic, but in general his view of logic is very similar to Boole's view of logic as the laws of though (see Boole 1854, Hailperin 1986, 1996). Now, again, this might tempt one to interpret Venn's view of the modalities as epistemic as Niiniluoto (1988b) does, but this is a rather uncharitable interpretation of Venn's work on logic. His work, by and large, predates the Fregean criticisms of psychologistic interpretations of logic, and so we can reasonably construe his view as the view that even the logical modalities are reducible to the probability calculus. This claim is least modestly supported in virtue of the fact that he is fully aware of the concept of mathematical certainty (see Venn 1866, p. 317) which appears to be an important precursor element of the logicistic interpretation of logic.

9 For formal reasons one might immediately regard this intuition regarding the structural similarity between possibilities and probabilities as flawed. If reduction requires isomorphism, then there must be a one-to-one mapping from the algebra of possibilities to the algebra of probabilities. But, isomorphisms are symmetric only if there is a one-to-one mapping between them (i.e. they are structural equivalencies and require that the mapping $F: A \rightarrow B$ matches unique elements in $A, x \in A$, with unique elements in $B, y \in B$, and that the inverse map $F^{-1}$ maps unique elements of $B$ into unique elements of $A$ ). As noted in note 3, there is no such mapping from the algebra of probabilities to the algebra of possibilities. Consequently, there can be no isomorphism between possibilities and probabilities. So, the Venn project can only be achieved if reduction does not require strict isomorphism, but rather some weaker relation like homomorphism, embedding or mere similarity. However, homomorphism, embedding and mere similarity are relations that are too weak to allow for eliminative reduction via definition. Homomorphisms are not symmetric, and, hence, although it is true that the algebra of possibilities is not homomorphic to the algebra of probabilities, it is true that the algebra of probabilities is homomorphic to the algebra of possibilities. This is interesting, because even if we relaxed the requirement for reduction from isomorphism to homomorphism, it would imply that modal eliminativism is false while leaving open the possibility of probabilistic eliminativism. But, this is no help to the nominalists and empiricists who want to eliminate the traditional modalities while retaining the concept of probability. 
logical possibility and whether it can be reduced to non-zero probability, the results achieved can be easily extended to necessity, equipossibility, etc. ${ }^{10}$ In any case, the identification of possibility with non-zero probability, and the subsequent elimination of possibility, will hold only if for any proposition $p$, possibly $p$ entails that $\mathrm{p}$ has a non-zero probability; formally, if $(\forall \mathrm{p})(\diamond \mathrm{p} \rightarrow P(\mathrm{p}) \neq 0)$ is true. ${ }^{11,12}$ However, given even a cursory analysis, the inference from possibly $\mathrm{p}$ to probably $\mathrm{p}$ appears as if it might be fallacious. In ordinary modal systems, one cannot infer an actuality from a mere possibility, and, hence, unless probabilities are not actualities the Venn project ought to be regarded with immediate suspicion. ${ }^{13}$ In fact, given a close analysis of Venn's views, Venn appears to want to eliminatively reduce the alethic modalities to empirically accessible physical properties. In fact, Venn (1866) originates the frequency interpretation of probability and it appears that Venn, like Reichenbach, regards probabilities as actual physical properties of the world on a par with other, more mundane, physical properties, and so Venn's tempered modal eliminativism is ultimately aimed at the implausible target of showing that modal propositions are about actual physical objects. However, in order to buttress these vague and intuitive objections about the inadequacy of Venn's project, it would be desirable to provide

10 It also remains to be seen whether or not the criticisms of the Venn project presented herein can be extended to weaker modal concepts. This question will be left open for future investigations, but if the criticisms can be extended to weaker modalities, then there might be interesting consequences with respect to views like those espoused by D. H. Mellor $(1995,2000)$. He claims, "The kind of necessity that " $c h_{\mathrm{c}}(\mathrm{E})=1$ " must express is usually if not always contingent: it can exist in our world without existing in all other possible worlds and so cannot be metaphysical necessity [1.6] ... But even if the necessity that " $c h_{\mathrm{c}}(\mathrm{E})=1$ " expresses is weaker than metaphysical necessity, it must still entail existence' (Mellor 1995, p. 31). So, although Mellor is rather unclear what kind of modalities are employed in formulating the necessity condition he uses to explain causation in Mellor 1995, if the modality in question is nonreducible to probability, then his view is in need of revision.

11 This must be the case because if $\neg(\mathrm{a} \rightarrow \mathrm{b})$, then it is possible that (a \& $\neg \mathrm{b})$, and, in the case at hand, this would entail that there can be logical possibilities which are not non-zero probabilities. But, the reductionistic cum eliminativist Venn project requires that all modalities, including possibilities, must be reducible to probabilities. Modalities and probabilities must be completely co-referential if they are identical, and so if there is some proposition that is possible but has a zero probability, then modalities and probabilities are not co-referential. See Levi 1980 for discussion of the incompatibility of such claims, and Zaman 1987 for an attempt to deal with the compatibility of such claims.

12 It is important to notice that the probability referred to in this expression is an unconditional probability, and one might be tempted to claim that all probabilities are conditional probabilities. Often the conditional aspect of probabilities is omitted in probability expressions for convenience, and so we must be clear about the conditional or unconditional nature of probabilities. Now, we do not want to bias the issue either way, and we will attempt to be clear when considering the various interpretations of the probability calculus. In brief, empirical versions of objectivist interpretations hold that probabilities are not conditional on any source of information (i.e. are not conditional in the usual sense), while both other types of interpretations generally regard probability statements as conditional. In any case, one might be tempted to claim that it is obvious that $\diamond \mathrm{p}$ is compatible with $P(\mathrm{p} \mid \mathrm{e})=0$, but that $\diamond(\mathrm{p} \mid \mathrm{e})$ is not obviously compatible with $P(\mathrm{p} \mid \mathrm{e})=0$. This suggestion brings to mind von Wright's suggestion noted above concerning treating all modalities as dyadic, and, more controversially, as treating the alethic modalities as conditional on tautologous evidence (see von Wright 1989, pp. 31-32). But, Venn does not appear to be aware of the concept of a conditional possibility, and is most concerned with the alethic modalities as opposed to, say, the epistemic modalities (which might, more plausibly, be regarded as conditional modalities). The view that the logical modalities can be seriously interpreted in conditional terms is unconvincing, and we will return to this topic in sections 2.1 and 2.2. But, Venn's project appears to be intended to eliminate logical possibility, etc., and not just epistemic possibility.

13 See van Fraassen 1989, Lemmon 1977, Konyndyk 1986 and Ackermann 1967 for discussions of permissible modal inferences and the problems associated with intensional logics. As Ackermann (1967, p. 17) points out, 'it is similarly clear that $\diamond p \rightarrow p$ cannot also be a theorem of a modal calculus without making $p$ and $\diamond p$ provably equivalent, thus making the modal operator $\diamond$ redundant.' 
a coherent argument for the conclusion that the Venn project cannot be successfully implemented.

\section{Interpretation of the probability calculus}

The probability calculus is constituted by three fundamental axiomatic claims that can be formulated as follows:

Axiom $1.0 \leqslant P(\mathrm{p}) \leqslant 1$ for all $\mathrm{p}$ in sample space $\mathrm{S}$.

Axiom 2. $P(\mathrm{~S})=1$.

Axiom 3. If $\mathrm{p}$ and $\mathrm{q}$ are mutually exclusive events, then $P(\mathrm{p} \cup \mathrm{q})=P(\mathrm{p})+P(\mathrm{q})$, and if $\mathrm{p}, \mathrm{q}, \mathrm{r}, \ldots$ is a finite or infinite sequence of mutually exclusive elements of $\mathrm{S}$, then $P(\mathrm{p} \cup \mathrm{q} \cup \mathrm{r} \cup \ldots)=P(\mathrm{p})+P(\mathrm{q})+P(\mathrm{r})+\ldots$

Here we will restrict our concerns to finite probability spaces that are continuous, although the main points made below can be extended to both the discrete and infinite cases. Note that Axioms 1-3 can be presented without anything like an intended interpretation, and the various interpretations of Axioms 1-3 can be viewed as different ways of characterizing the elements of $S$ and the nature of the probability function or probability distribution over S. In any case, there are two general classes of interpretations of the probability calculus: (1) subjective interpretations (SIs); and (2) objective interpretations (OIs). The former class includes subjective Bayesianism, de Finetti's theory, F.P. Ramsey's theory, L.J. Savage's theory, etc., and the latter class includes Popper's propensity interpretation, Carnap's and Keynes' logical theories of probability, ${ }^{14}$ frequency interpretations like those of Reichenbach and von Mises, etc.

The distinction divides the various interpretations of A1-A3 into those that claim that probabilities are epistemic, and those which hold that probabilities are, in some way, independent of epistemic states. Other ways of classifying interpretations of the probability calculus are typically more fine-grained, but this broad distinction (which appears to be exhaustive) will serve our purposes here. ${ }^{15}$ What will be argued for here is that given any interpretation of the probability calculus, possibly $\mathrm{p}$ does not entail that $\mathrm{p}$ has a non-zero probability, and, as a result, the Venn project is fundamentally misguided. The main argument will have the form of an argument by cases with the conclusion that the conjunction of possibly $\mathrm{p}$ and the probability of $\mathrm{p}$ equals zero is consistent, or, formally, that $\diamond \mathrm{p} \& P(\mathrm{p})=0$ can be true. ${ }^{16}$ This constitutes a counterexample to the claim that possibly p entails that the probability of $\mathrm{p}$ is non-zero. If this can be proved, then it follows that it is $\mathrm{p}$ the case that possibly $\mathrm{p}$ entails that the probability of $\mathrm{p}$ is non-zero, and, consequently, that possibility cannot be reduced to probability as the Venn project would have it. Clearly the bona fides of this argument hangs on providing some justifications for the claims that given SI 'possibly p' and

14 As we shall see later, there is some question whether the logical theory of probability ought to be regarded as a species of the subjectivist theories or as a species of the objectivist theories. Ultimately it is probably best regarded as a species of OI.

15 For a survey of various ways of classifying interpretations of the probability calculus, and discussion of their adequacy see Weatherford (1982, ch. 1). In terms of Weatherford's four fold distinction of theories of probability, OI would include the relative frequency (RF) and a priori (AP) theories, and SI would include the classical (CTP) and subjective (SUB) theories.

16 For the sake of simplicity assume that $P(\mathrm{p})$ is definable, either as an absolute probability or as a conditional probability, but in general any reference to evidence and background knowledge will be omitted throughout. 
'the probability of $\mathrm{p}$ is zero' can be consistent and that given OI 'possibly p' and 'the probability of $\mathrm{p}$ is zero' also can be consistent.

\section{Subjectivist interpretations ${ }^{17}$}

Are there good reasons for accepting that subjectivist interpretations of the probability calculus entail the consistency of possibly $p$ and that the probability of $p$ is zero? Given subjective interpretations of the probability calculus, probabilities are identified as degrees of belief or credal probabilities. Hence, probabilities are clearly epistemological. So, subjective probabilities are typically regarded as confidence levels with respect to the proposition in question relative to some source of background information. As such, the subjectivist typically regards all probabilities as conditional probabilities, conditional on one's background beliefs. One's totality of probability assignments over one's set of beliefs is the probability function that one adopts, and typical subjectivists argue that there is no objectively correct probability function, or probability distribution, that should be adopted. Importantly, many (if not most) subjectivists reject limiting acceptable probability distributions to regular distributions; i.e. those that are strictly coherent.

Strict coherence amounts to the claim that $P(\mathrm{p})=0$ if and only if $\mathrm{p}$ is logically false. In other words, a distribution is strictly coherent if $P(\mathrm{p})=0$ if and only if $\mathrm{p}$ is logically impossible. Insofar as subjectivists only require that probability distributions be coherent and not strictly coherent, on this type of view it seems obviously consistent that a proposition may be logically possible while that proposition has a probability of zero relative to background information. Rejecting strict coherence as a limitation on probability distributions amounts to the claim that such situations are consistent. For example, it appears to be consistent that some event is a logical possibility while it has a zero probability relative to some system of background information. Some state of affairs may not be logically false, but the evidence available to some cognizer may prompt her to assign a zero probability to that state of affairs. So, on subjectivist interpretations of the probability calculus it clearly seems possible that there are logical possibilities with zero probabilities. It seems that, given SI, $(\diamond p \& P(p)=0)$ can be true, and this seems to be a direct result of the typical subjectivists rejection of strict coherence. But, can one simply reject strict coherence as too strong an imposition on admissible probability distributions without further comment? Perhaps the subjectivists' rejection of strict coherence is unwarranted. So, what are the typical justifications for strengthening the probability calculus to make it strictly coherent? If there are no good independent reasons to require that strict coherence ought to be adopted, then there will be no non-question begging reasons to claim that the logical possibility of $p$ is incompatible with the subjective assignment of a zero probability to $\mathrm{p}$.

The standard Dutch book justifications of mere coherence depend on the concept of a fair bet, and presume, rightly, that it would be irrational for someone to enter into a betting situation that ensured that they would suffer a loss in the long run. The standard justification of why we ought to accept these principles of rational behavior seem to depend on intuition and/or consider the coherence

17 I include discussion of subjective interpretations for the sake of both comprehensiveness and generality. In point of fact, Venn himself rejected subjective interpretations of the probability calculus, like that offered by De Morgan, as is clear from his comments (1962, pp. ix, 119n, 122-3). Nevertheless, as my aim to show the inadequacy of all forms of tempered modal eliminativism and not just the inadequacy of the Venn project, it is necessary to address such interpretations. 
condition to be analytic. ${ }^{18}$ But, this is rather presumptuous to say the least, and appears to beg the question, 'Is the claim that $P(\mathrm{p})=0$ if and only if $\mathrm{p}$ is logically impossible analytic in any understandable sense of the term 'analytic' such that it is on a par with simple mathematical truths'? Perhaps, but intuitions may, and do, differ concerning this issue. For example, De Finetti, Ramsey, and, recently, Levi all urge us to reject strict coherence; i.e. to reject the claim that probability distributions must be regular. ${ }^{19}$ Their reasons for rejecting strict coherence, however, are not on a par with reasons that one might give for rejecting the putatively analytic claim that ' $2+2=5$ ', and this suggests that the criterion may not really be an analytic claim. Their reasons for rejecting the criterion of strict coherence are founded upon differing intuitions about what possible betting situations ought to be characterized as rational, and it is not at all clear that labeling the criterion 'analytic' provides anything like a justification of that condition. In fact, with the advent of naturalistic theories of rationality and Quine's demolition of the analytic/synthetic distinction I would be inclined to believe that principles about what constitutes rational human betting behavior are empirical claims that are neither analytic nor knowable $a$ priori. ${ }^{20}$ So, the consistency claim holds with respect to subjective interpretations of the probability calculus unless there is some non-question begging justification of strict coherence and it does not seem to be the case that there is such a justification available for strict coherence.

\section{Objectivist interpretations}

Are there reasons for accepting the claim that objectivist interpretations of the probability calculus entail the consistency of possibly $p$ and that $p$ has a probability of zero? As suggested above justifying this claim is a bit more difficult than justifying the same claim modulo the interpretation as subjective, and this is, in part, due to the wide variety of objectivist interpretations of the probability calculus. In order to simplify the discussion the class OI needs to be partitioned into those objectivist interpretations that are empirical interpretations, $\mathrm{OI}_{\mathrm{E}}$, and those objectivist interpretations that are logical interpretations, $\mathrm{OI}_{\mathrm{L}}$. It is the latter that will pose the most difficult problem for establishing the main argument and rejecting the Venn project. First, however, we must consider $\mathrm{OI}_{\mathrm{E}}$.

On typical empirical or physicalist versions of the objectivist interpretations of the probability calculus probabilities are regarded as properties of event types or event tokens, or, perhaps, as dispositional properties of physical objects. ${ }^{21}$ For the sake of brevity, in what follows I will simply refer to event types, and assume that the results can be applied mutatis mutandis to varieties of this class of theories that regard probabilities as properties of event tokens or physical objects. Given these empirically based theories of the probability calculus, the probability of an event type depends on the facts of the matter at some particular world region. But, such probabilities do not make reference to any evidence whatsoever, and, hence, in this case there is no worry

18 See Shimony (1955, pp. 129-30).

19 See De Finetti 1972, Ramsey 1926 and Levi 1978.

20 For some more or less compelling examples of such views see Kahneman et al. 1982, Cherniak 1986, Kitcher 1992, Smokler 1990 and Stich 1990. And Quine's classic criticism of the analytic/synthetic distinction is found in Quine 1961. For some objections to this view of the status of principles of rationality see Biro and Ludwig 1994 and Evnine 2001.

21 The latter consideration is necessary to cover Popper's propensity interpretation of probabilities. On Popper's theory of probability probabilities are dispositions of physical objects. 
about whether probabilities are conditional probabilities and there is no worry about whether or not the probability calculus should be limited by the imposition of strict coherence. On such theories probabilities are simply objective properties of the world just like any other physical property. If this is so, then it may be the case that some event is logically possible while it has a zero probability in that region of that world. For example, it may be the case that it is logically possible that electrons or the $Z^{0}$, the intermediate vector boson, can decay, but as it stands, it is an empirical fact that neither type of particle ever decays in that region, where the region may be the totality of the world. So, although the event type electron decay is a logical possibility in this region (it is not a logical falsity) it may have a zero probability as determined by the facts of our world. It is, in fact, the case that events types of these two sorts are not permitted by the actual laws of our world, and (at least intuitively) it would seem to be the height of irrationality to claim that such events have some non-zero probability nonetheless. The corresponding region in some other possible world, governed by more or less different physical laws, may be physically compatible with such event types, but that such events are consistent with some set of counter-factual physical laws that can be formulated (i.e. are permitted by some other set of logically consistent laws) does not seem to indicate that the probability of such events in the actual world is non-zero. Take the typical frequency interpretation of probabilities. ${ }^{22}$ An event type, or a sequence of type-similar events, is probable in virtue of the fact that it happens with some frequency or other in the limit case, i.e. some chance set-up occurs with some frequency in the limit, and there are objective facts about the limit frequency of these types of events just like there are facts about any other type of physical event. So, on this interpretation of the probability calculus:

$$
P(\mathrm{p} \mid \mathrm{q})=\underset{n \rightarrow \infty}{\text { Limit }} f r_{n}(\mathrm{p} \mid \mathrm{q})
$$

Where the frequency of an event is defined as:

$$
f r_{n}(\mathrm{p} \mid \mathrm{q})=\frac{f r_{n}(\mathrm{p} \bullet \mathrm{q})}{f r_{n}(\mathrm{q})}
$$

in short, the frequency of $\mathrm{p}$ given $\mathrm{q}$ is the ratio of the number of individuals or events having both $\mathrm{p}$ and $\mathrm{q}$ divided by the number of individuals or events having $\mathrm{q}$. Where $\mathrm{q}$ holds for all $p$, the absolute probability of $p$ is just the limit frequency of $p$. The sense in which such probabilities are conditional is not intended to be conditional on any background belief system. In any case, many more types of chance set-ups or event types are logically possible than actually ever occur, and if this is so, then it would seem obvious that there can be logical possibilities which have zero probabilities. For example, the limit frequency of electron decay in the actual world is 0 relative to electron set-ups and yet it seems manifestly clear that it is logically possible that electron decay could occur. The general reason why this appears to be so is that on empirical versions of the objectivist interpretation of the probability calculus, prob-

22 Interestingly, as noted in the introduction, Venn has been credited (along with Peirce) as the founder of the relative frequency interpretation of probabilities, and this view is proposed in Venn 1866. Consequently, one might plausibly interpret Venn's modal eliminativism more specifically as the claim that all the modalities can be eliminatively reduced to physical probabilities. However, this more controversial assumption is not presupposed here. 
abilities depend on the vagaries of the facts while logical possibilities are necessarily independent of any factual, empirical, considerations. It seems to be the case by definition empirical probabilities of logically possible event types that never occur will be 0 . So, it seems that $\mathrm{OI}_{\mathrm{E}}$ permits $(\diamond \mathrm{p} \& P(\mathrm{p})=0)$ to be true.

What of logical versions of the objectivist interpretation of the probability calculus such as Carnap's? On $\mathrm{OI}_{\mathrm{L}}$ can $(\diamond \mathrm{p} \& P(\mathrm{p})=0)$ be true? In order to answer this question we need to examine just what the logical theory claims probabilities are, and how the theory differs from both the empirical versions of the objectivist interpretation of the probability calculus and from subjectivist interpretations of the probability calculus. Carnap's (1962) explications of the meaning of logical probability, probability $_{1}$, appear to be ambiguous between a kind of inter-subjectivism and a kind of objectivism of the sort in which we are interested in this section. ${ }^{23}$ In the preface to the second edition of The Logical Foundations of Probability Carnap says,

(probability ${ }_{1}$ ) was informally explained in $\S 41$ in several ways: (a) as the degree to which a hypothesis $h$ is confirmed or supported by the evidence $e$; (b) as a fair betting quotient; and (c) as an estimate of relative frequency. Even at that time I regarded (a) as less satisfactory than (b) or (c); today I would avoid formulations of the kind (a) because of their ambiguity (see Points B and C below). Although the concept of logical probability in the sense here intended is a purely logical concept, I think that the meaning of statements like "the probability of $h$ with respect to $e$ in $2 / 3^{\prime}$ can best be characterized by explaining their use, in combination with the concept of utility, in the rule for the determination of rational decisions. (1962, p. xv.)

Carnap explicitly claims that (a) is ambiguous in a well-discussed sense, ${ }^{24}$ but it seems that (a), (b) and (c) also introduce ambiguity into the concept of logical probability in an important, but different, sense. This ambiguity centers on whether or not logical probabilities are actually subjective or objective, and this ambiguity is revealed in examining some of the various ways in which subsequent authors have characterized the logical theory of probability. On the one hand, explicating logical probability as something like (c), as an estimate of relative frequency, seems to introduce an unacceptable element of subjectivism into the supposedly logical concept of probability. However, ignoring (c) as too apt to be interpreted subjectively, and focusing on explication (a) or (b) might still lead one to believe that logical probabilities are not really objective in any real sense.

One might be tempted to regard the logical theory as a species of the objectivist theory of probability as it was seemingly intended to be. However, given some characterizations of logical probability, one might also be tempted to regard it as an inter-subjectivist theory of probability. ${ }^{25}$ As such, it might seem plausible to assert

23 Hacking notes this ambiguity in Hacking (1975, p. 149), and indicates that it is often presented as a criticism of logical theories of probability. Also, Jeffery 1975 acknowledges the ambiguous sense in which Carnap's theory of confirmation is meant to be logical.

24 See Carnap (1962, pp. xv-xix) for an explanation of the ambiguity associated with the (a) explication.

25 This kind of theory is explicitly defended by Donald Gillies (1991), and Gillies argues that inter-subjective interpretations of probabilities are closely related to subjectivist interpretations of probabilities. On his view probability distributions can be attributed to social groups in states of consensus that are informationally transparent rather than to individuals, as in standard subjective interpretations. But, this view turns out to depend on contingent features of such social groups, and is equally susceptible to the criticisms directed at purely subjectivist interpretations of the probability calculus. 
that it really is just a type of subjectivist theory, especially when we recognize that the characterization of the logical theory given above appeals to the contingent epistemological structure of how humans think. If this is the case, however, then, with only as little modification, the logical theory could be shown to permit $\diamond \mathrm{p} \& P(\mathrm{p})=0)$ to be true for roughly the same reasons that SI does. Where in the case of subjectivist theories we appealed to the variations in probability assignments across agents in order to justify the consistency claim concerning zero probabilities and logical possibilities, in the case of the inter-subjectivist theory we can appeal to possible variations across kinds of cognitive agents. It seems perfectly consistent to assert that some suitably different cognitively endowed entity assigns a zero probability of such a thing occurring and it is obviously logically possible that such an event obtains. The problem with the inter-subjectivist theory, as characterized by Weatherford, is that it turns out that it does appeal to empirical matters in a more subtle way. Specifically, the inter-subjectivist theory appeals to the de facto structure of how we think, to our actual epistemological constitution, and it is certainly possible that other cognitively endowed entities do not share our cognitive structure. ${ }^{26}$ So, if the logical theory is regarded as this kind of anthropomorphic subjectivist theory, then it permits $(\diamond \mathrm{p} \& P(\mathrm{p})=0)$ to be true.

On the other hand, if, as suggested at the outset, one jettisons the appeal to the facts concerning how we think in characterizing the logical theory of probability, and regard the logical theory as a species of objectivist theory, then can we show that on $\mathrm{OI}_{\mathrm{L}}(\diamond \mathrm{p} \& P(\mathrm{p})=0)$ can be true? If one can, then the main argument presented in $\$ 2.0$ will have been established as sound. So, how are we to think of probabilities in the logical theory if they are not subjective in any sense? In a typical presentation of the view, F.C. Benenson tells us that the logical view of probability,

... is the view that probability judgments are always relative to the evidence, and in fact merely indicate the degree of certainty a particular body of evidence gives to the hypothesis we are concerned with. (1984, pp. 13-14.)

Moreover, he adds that,

according to the theory, it is not merely such evidence [empirical evidence] which permits us to know the truth of ordinary probability statements, but such evidence combined with a single logical relation of partial entailment it bears to the hypothesis in question, provides us with an effective method for determining the truth of the statement on the probability of that hypothesis - we simply perform the finite task of contemplating that evidence, and then, to determine the truth of the statement, effectively compute the degree of logical probability the evidence gives the hypothesis. (1984, p. 17.)

On this sort of interpretation, the degree of support that a body of evidence gives to an hypothesis via their logical relation seems to be purely objective and clearly conditional, and this characterization seems to correspond most closely to Carnap's

26 The point here is reminiscent of the point made concerning Kant's forms of sensible intuition as presented in The Critique of Pure Reason. The forms of sensible intuition, space and time, are regarded as necessary for experience, but the necessity involved here is relative and not absolute. The forms of sensible intuition may not hold objectively for all kinds of cognitive beings. 
(a) explication of the logical concept of probability. The propositions that constitute the body of evidence entail, or partially entail, the hypothesis, and so allow us to attribute a probability to the hypothesis relative to the evidence in accord with the axioms of the probability calculus. But the (a) explication is rejected by Carnap, and he seems to favor the (b) explication, what Hilpinen has labeled the belief-explication of logical probability. ${ }^{27}$ However, it is far from obvious that the (b) explication can be plausibly interpreted as being objective, especially because it seems to be intimately related to the concept of rational decision-making. If one interprets probabilities in terms of rational (human) decision-making, then it appears as if one might again be confronted with a subjectivist characterization of logical probability like that referred to above, and thus the argument in $\$ 2$ would turn out to be sound.

One might simply claim, however, that Carnap's fair betting quotients make no appeal to subjective states at all. Rather, probabilities might be interpreted objectively as being defined in terms of fair betting quotients; i.e. as being an objective fragment of logic or mathematics. The various Dutch book theorems aim to show that following the axioms of the probability calculus define the concept of a fair bet, and this seems to capture the meaning that Carnap's (b) interpretation of logical probability is intended to capture without falling prey to subjectivism. ${ }^{28}$ But, on such an objective reading of logical probability does $\mathrm{OI}_{\mathrm{L}}$ permit $(\diamond \mathrm{p} \& P(\mathrm{p})=0)$ to be true? It turns out that there are many cases in which it will. Consider Olbers' paradox in cosmology. It is logically possible that our universe is a steady state universe, and, under certain well-known conditions, if the universe were a steady state universe, then the sky would not be dark at night. However, the sky is dark at night, and so the steady state theory is empirically falsified. In such a case it is clearly possible that one might regard the steady state universe hypothesis as logically possible, yet as having a conditional probability of 0 . But, we need not consider only cases where some theory has been refuted or falsified. Cases of the sort with which one is here interested may also result from limited evidence. It is logically possible that tachyons exist, but it might be the case that the hypothesis that tachyons exist has a zero probability on the available evidence or relative to some specified data set. Even if one accepts the requirement of total evidence, total empirical evidence, it will can be the case that there will be logically possible hypotheses that gain no probabilistic support from the empirical evidence (i.e. their conditional probabilities relative to evidence $e$ will be zero). In fact, given the totality of empirical evidence it appears as if it might be the case on the logical theory that no false but logically possible theory has a non-zero probability. One might think that inductivists who adopt the logical interpretation of probability hold that all of the evidence would exhaustively specify all and only truths. ${ }^{29}$ Every hypothesis not supported by the totality of evidence must have probability zero, even if it is a logically possible state of affairs. For example, the totality of evidence may make it absolutely, and objectively, certain that electrons do not decay (i.e. that the probability of electron decay is, in fact, zero), while it is certainly logically possible that electron decay occurs (i.e. the totality of evidence, were it different, may have made it certain that electron decay occurs).

27 See Hilpinen 1975.

28 Although this appears to be the intended interpretation of logical probabilities, it is not at all clear that such a concept makes sense. The empirical nature of what constitutes rational human behavior appears to undermine the possibility of grounding such putatively logical concepts.

29 Of course this presumes that we are talking about empirical claims, and that there really is one true description of reality. It may turn out, if the instrumentalists are correct and induction is unwarranted, that a full specification of the empirical facts would be compatible with several theories. This complication will be ignored here, however. 
However, typical advocates of the logical theory adopt the criterion of strict coherence, and as one saw above, strictly coherent probability distributions are those in which the only propositions to which zero probabilities can be assigned are those propositions that are logically contradictory. Must the advocate of the logical interpretation of probabilities accept the imposition of strict coherence on the probability calculus? Again, if there are no good reasons independent reasons to do so, then there will be no non-question begging reason why there cannot be logical possibilities with zero probabilities. As Ramsey and others have argued coherence is forced on us by examining the axioms of the probability calculus in light of rational betting behavior such that one would not put oneself in a situation in which one is guaranteed to loose in the long run. But, strict coherence is not related to the probability calculus in the same way. Strict coherence suggests that it is merely irrational to place oneself in a betting situation not only that is guaranteed to produce a loss, but also that it is irrational to put oneself in a betting situation guaranteed to make one break even and face a loss in one possible world. ${ }^{30}$ It is not clear that Dutch book arguments can legitimately justify the adoption of strict coherence in the same way that they can justify unrestricted coherence, as such a Dutch book argument necessitates strengthening the probability calculus in accord with more controversial intuitions concerning rational betting behavior. If one is attempting to justify the probability calculus why should one require admissible probability distributions to be strictly coherent, and, hence, require that the probability calculus be modified? Again, as pointed out in the context of subjectivist interpretations of the probability calculus such justifications as are available depend on intuitions and rather suspicious claims about the analyticity of the strict coherence criterion, and this feeble justificatory facade begs the question against those who do not share such intuitions. So, $\mathrm{OI}_{\mathrm{L}}$ does appear to permit $(\diamond \mathrm{p} \& P(\mathrm{p})=0)$ to be true, whether it is characterized as subjective or as objective provided there are no non-question begging justification of strict coherence and there do not seem to be any such justification currently available.

\section{Conclusion}

The counter-examples presented in $\$ 2$ show that the main argument given herein is sound. Thus, $(\diamond \mathrm{p} \rightarrow P(\mathrm{p}) \neq 0)$ appears to be false, and so it appears to be the case that, for conceptual reasons, possibility cannot be reduced to probability under any sort of interpretation of the probability calculus despite the appeal that such an eliminative reduction would have for empiricists. Consequently, it would seem that we can, at least tentatively, conclude that it is a conceptual truth that the Venn project, tempered modal eliminativism, is fundamentally misguided. As such, it is reasonable to believe that the concepts of the alethic modalities are in no danger of being reduced to probabilities, despite this deeply interesting, truly innovative and initially promising suggestion from John Venn. ${ }^{31}$ As such, if empiricists are to lay the ghost of modality to rest, they must seek some other more effective stratagem to do so.

30 See Shimony (1955, p. 127).

31 Curiously, the Leibnizian program of probabilistic eliminativism is also fundamentally misguided (although, formally, it is in better shape), but that is a topic for another paper. Presuming that this is the case, however, rejection of both the Venn project (modal eliminativism) and the Leibnizian project (probabilistic eliminativism) would show that the concepts of probability and of the traditional modalities are simply distinct. 


\section{References}

Ackermann, R. 1967. Introduction to Many Valued Logics, London: Routledge \& Kegan Paul.

Benenson, F. C. 1984. Probability, Objectivity and Evidence, London: Routledge \& Kegan Paul.

Biermann, K.-R. and Faak, M. 1957. 'G. W. Leibniz De incerti aestimatione', Forschungen und Fortschritte, 39, 142.

Bigelow, J. C. 1976. 'Possible Worlds Foundations for Probability', Journal of Philosophical Logic, 5, 299320.

Biro, J. and Ludwig, K. 1994. 'Are There More than Minimal A Priori Limits on Irrationality?', Australasian Journal of Philosophy, 72, 89-102.

Boole, G. 1854. An Investigation of the Laws of Thought, New York: Dover Publications.

Carnap, R. 1962. The Logical Foundations of Probability, 2nd edn, Chicago: University of Chicago.

Cherniak, C. 1986. Minimal Rationality, Cambridge: MIT Press.

De Finetti, B. 1972. Probability, Induction and Statistics, New York: Wiley.

Evnine, S. 2001. 'The Universality of Logic: On the Connection Between Rationality and Logical Ability', Mind, 110, 335-67.

Gillies, D. 1991. 'Intersubjective Probability and Confirmation Theory', British Journal for the Philosophy of Science, 42, 513-533.

Hacking, I. 1971. 'The Leibniz-Carnap Program for Inductive Logic', Journal of Philosophy, 68, 597-610.

Hacking, I. 1975. The Emergence of Probability, Cambridge: Cambridge University Press.

Hailperin, T. 1986. Boole's Logic and Probability, 2nd edn, New York: North-Holland.

Hailperin, T. 1996. Sentential Probability Logic, Cranbury, NJ: Associated University Presses.

Hilpinen, R. 1975. 'Carnap's New System of Inductive Logic', in J. Hintikka, Rudolph Carnap, Logical Empiricist, Dordrecht: D. Reidel, 330-352.

Jeffery, R. 1975. 'Carnap's Inductive Logic', in J. Hintikka, Rudolph Carnap, Logical Empiricist, Dordrecht: D. Reidel, 325-332.

Kahneman, D., Slovic, P. and Tversky, A. 1982. Judgment Under Uncertainty: Heuristics and Biases, Cambridge: Cambridge University Press.

Kitcher, P. 1992. 'The Naturalist's Return', The Philosophical Review, 101, 53-114.

Konyndyk, K. 1986. Introductory Modal Logic, Notre Dame, Indiana: University of Notre Dame Press.

Lemmon, E .J. 1977. The 'Lemmon Notes': An Introduction to Modal Logic. American Philosophical Quarterly Monograph Series, Monograph No. 11, Oxford: Blackwell.

Levi, I. 1978. 'Coherence, Regularity and Conditional Probability', Theory and Decision, 9, 1-15.

Levi, I. 1980. The Enterprise of Knowledge, Cambridge: MIT Press.

Mellor, D. H. 1995. The Facts of Causation, London: Routledge.

Mellor, D. H. 2000. 'Possibility, Chance, and Necessity', Australasian Journal of Philosophy, 78, 16-27.

Niiniluoto, I. 1988a. 'From Possibility to Probability: British Discussions on Modality in the Nineteenth Century', in S. Knuuttila, Modern Modalities: Studies in the History of Modal Theories from Medeival Nominalism to Logical Positivism, Dordrecht: Kluwer, 275-310.

Niiniluoto, I. 1988b. 'Probability, Possibility, and Plenitude', in J. Fetzer, Probability and Causality, Dordrecht: D. Reidel, 91-108.

Nilsson, N. J. 1986. 'Probabilistic Logic', Artificial Intelligence, 28, 71-87.

Quine, W. V. O. 1947. 'The Problem of Interpreting Modal Logic', Journal of Symbolic Logic, 12, $43-48$.

Quine, W. V. O. 1953. 'Three Grades of Modal Involvement', Proceedings of XIth International Congress of Philosophy, vol. 14.

Quine, W. V. O. 1960. Word and Object, Cambridge: MIT Press.

Quine, W. V. O. 1961. 'Two Dogmas of Emiricism', in W.V.O. Quine, From a Logical Point of View, 2nd edn., Cambridge: Harvard University Press, 20-46.

Ramsey, F. P. 1926. 'Truth and Probability', in D. H. Mellor, Philosophical Papers, Cambridge: Cambridge University Press, 52-99.

Shimony, A. 1955. 'Coherence and the Axioms of Confirmation', Journal of Symbolic Logic, 20, 1-28.

Smokler, H. 1990. 'Are Theories of Rationality Empirically Testable?', Synthese, 82, 297-306.

Stich, S. 1990. The Fragmentation of Reason, Cambridge: MIT Press.

Van Fraassen, B. 1977. 'The Only Necessity is Verbal Necessity', Journal of Philosophy, 74, 71-85.

Van Fraassen, B. 1980. The Scientific Image, Oxford: Clarendon Press.

Van Fraassen, B. 1989. Laws and Symmetry, Oxford: Clarendon Press.

Venn, J. 1866. The Logic of Chance, London: MacMillan.

Wright, G. H., von. 1989. 'Intellectual Autobiography of Georg Henrik von Wright', in P. A. Schilpp and L. E. Hahn, The Philosophy of Georg Henrik von Wright, La Salle: Open Court, 1-55.

Weatherford, R. 1982. Philosophical Foundations of Probability, London: Routledge \& Kegan Paul.

Zaman, A. 1987. 'On the Impossibility of Events of Zero Probability', Theory and Decision, 23, 157-9. 
Copyright of History \& Philosophy of Logic is the property of Taylor \& Francis Ltd and its content may not be copied or emailed to multiple sites or posted to a listserv without the copyright holder's express written permission. However, users may print, download, or email articles for individual use. 